\title{
Synthetic Extracellular Matrices as a Toolbox to Tune Stem Cell Secretome
}

Kaizheng Liu, Tomas Veenendaal, Maury Wiendels, Alejandra M. Ruiz-Zapata, Justin van Laar, Rafail Kyranas, Hilde Enting, Bram van Cranenbroek, Hans J. P. M. Koenen, Silvia M. Mihaila,* Egbert Oosterwijk, and Paul H. J. Kouwer*

Cite This: ACS Appl. Mater. Interfaces 2020, 12, 56723-56730

Read Online

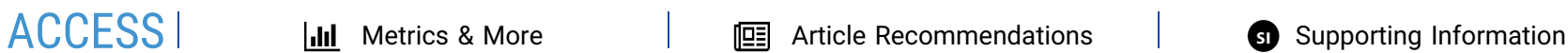

ABSTRACT: The application of stem cell-derived secretome in regenerative therapies offers the key advantage that instead of the stem cells, only their effective paracrine compounds are in vivo delivered. Ideally, the secretome can be steered by the culture conditions of the stem cells. So far, most studies use stem cells cultured on stiff plastic substrates, not representative of their native $3 \mathrm{D}$ environment. In this study, cells are cultured inside synthetic polyisocyanide (PIC)-based hydrogels, which are minimal, tailorable, and highly reproducible biomimetic matrices. Secretome analysis of human adipose-derived stem cells (multiplex, ELISA) displays that matrix manipulation is a powerful tool to direct the secretome composition. As an example, cells in nonadherent PIC gels secrete increased levels of IL-10 and the conditioned media from 3D culture accelerate wound closure. In all, our PIC-based approach opens the door to dedicated matrix design to engineer the secretome for custom applications.

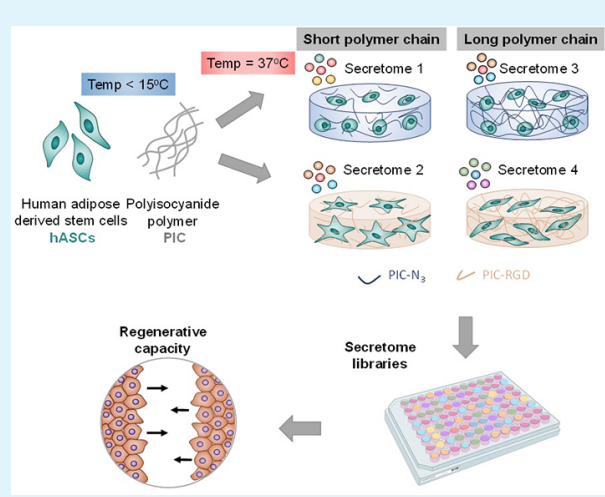

KEYWORDS: synthetic extracellular matrices (ECM), stem cell secretome, polyisocyanide hydrogels, interleukin-10 (IL-10), wound healing

\section{INTRODUCTION}

The stem cell secretome is attracting an increasing amount of attention due to its use in cell-free regenerative therapies. ${ }^{1}$ Experimental studies show that the regenerative capacity of human adipose-derived stem cells (hASCs) is likely to be dependent on the paracrine activity and less so on their engraftment, ${ }^{2-4}$ which suggests that therapies in regenerative medicine can be implemented through tissue conditioning without cell implantation. The stem cell secretome is defined as the collection of soluble paracrine factors secreted by stem cells. These mixtures include cytokines, chemokines, cell adhesion molecules, growth factors, hormones, exosomes, microvesicles, serum and extracellular matrices (ECM) proteins, proteases, and lipid mediators. ${ }^{1,5}$ This complex mixture influences cellular functions such as proliferation, differentiation, and communication ${ }^{6}$ and is an important contributor to therapeutic processes such as wound recovery and wound healing. ${ }^{3,7,8}$ Ideally, one would like to optimize the components in the secretome toward specific applications.

Conditioned media (CM) are media that are harvested from cultured cells after a specified period. Many groups started studying CM collected from cells cultured on two-dimensional (2D) substrates 9,10 and also demonstrated that the culture dimension $^{11,12}$ impacts on the outcome: different culture dimension conditions correlated with changed cell functions, ${ }^{13}$ including paracrine activities. CM studies from 3D grown cells are scarce, although the $3 \mathrm{D}$ environment represents the natural niche of (stem) cells. ${ }^{14}$ So far, the studies have focused primarily on matrices of natural polymer gels, ${ }^{11}$ on cell aggregates, ${ }^{15}$ on their combinations, ${ }^{16}$ or on electrospun fiber scaffolds. ${ }^{17}$ This approach is hampered by the use of natural biomatrices that often contain multiple partly defined components, including growth factors and cytokines. As such, the role of the matrix properties in the modulation of paracrine factors remains unclear also due to the lack of tunability of matrices used in the research.

Synthetic matrices, in contrast to their biological counterparts, can be more freely designed. The molecular structure, functionalization, and gel architecture can be used to manipulate (stem) cell behavior. ${ }^{18}$ We hypothesize that the stem cell secretome can be tuned by culture in different 3D synthetic matrices. As a model, we employed the synthetic polyisocyanide (PIC) hydrogel with fully controllable properties. The fibrous structure provides the gels with the porous

Received: September 8, 2020

Accepted: November 12, 2020

Published: December 11, 2020 
Table 1. PIC Hydrogels Used for Stem Cell Encapsulation ${ }^{e}$

\begin{tabular}{|c|c|c|c|c|c|c|c|}
\hline hydrogels & polymers & $M_{\mathrm{v}}(\mathrm{kg} / \mathrm{mol})^{a}$ & $L_{\mathrm{c}}(\mathrm{nm})^{b}$ & $c\left(\mathrm{mg} / \mathrm{mL}^{-1}\right)^{c}$ & $D(\mu \mathrm{M})^{d}$ & $G^{\prime}(\mathrm{Pa})$ & $\sigma_{\mathrm{c}}(\mathrm{Pa})$ \\
\hline S1 & P1-N 3 & 398 & 158 & 1 & & 141.1 & 10.6 \\
\hline S2 & P1-RGD & 398 & 158 & 1 & 28.5 & 62.8 & 7.7 \\
\hline S3 & P2- $\mathrm{N}_{3}$ & 576 & 229 & 1 & & 325.3 & 29 \\
\hline S4 & P2-RGD & 576 & 229 & 1 & 28.5 & 181.9 & 19.9 \\
\hline
\end{tabular}

${ }^{a} M_{\mathrm{v}}=$ viscosity-based molecular weight of azide-appended polymers. ${ }^{b}$ Average contour length based on $M_{\mathrm{v}} .{ }^{c}$ Polymer concentration. ${ }^{d}$ Density of conjugated RGD peptide. ${ }^{e}$ Molecular weights of precursor PIC-N $\mathrm{N}_{3}$ polymers were measured. The mechanical properties of S1 and S3 have been measured in triplets and show overlapping curves (Figure S1).

a

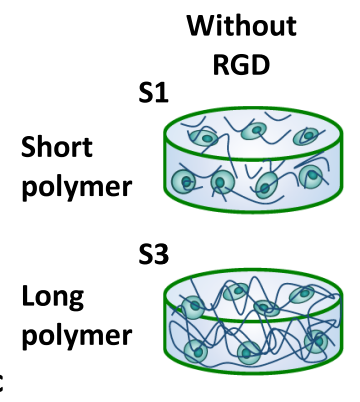

b

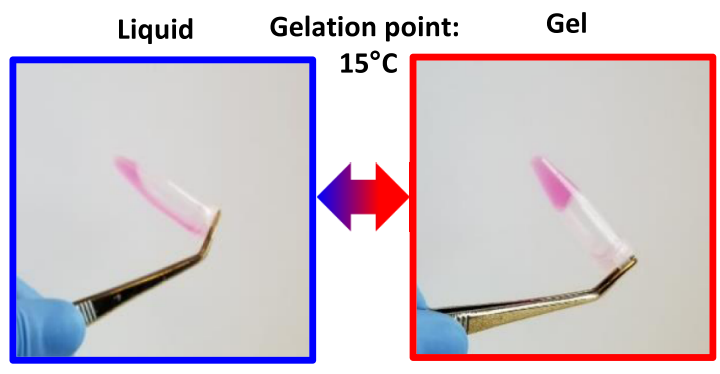

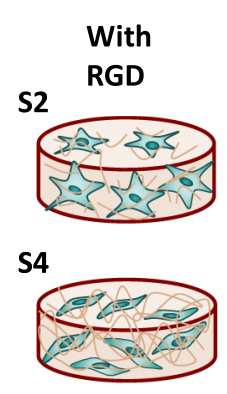

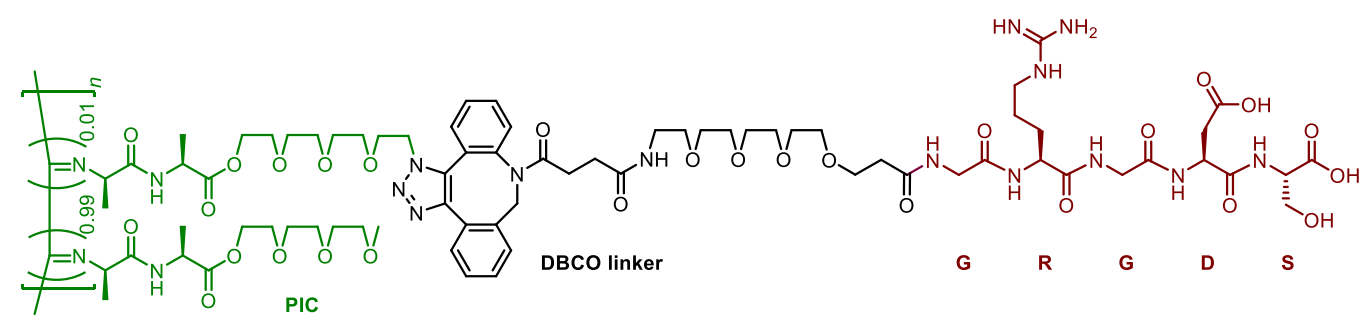

d

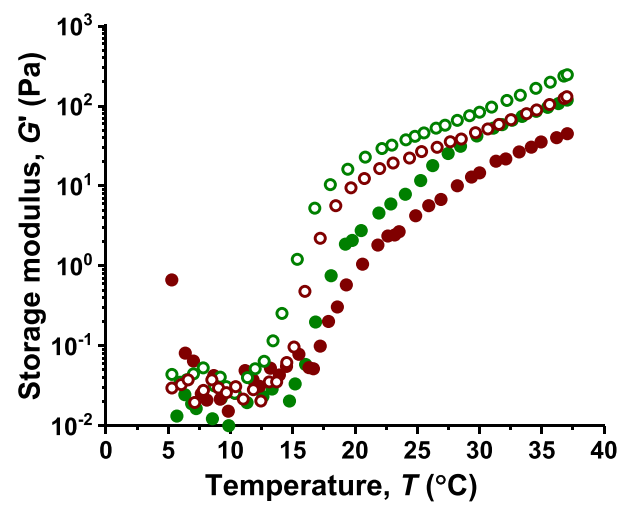

e

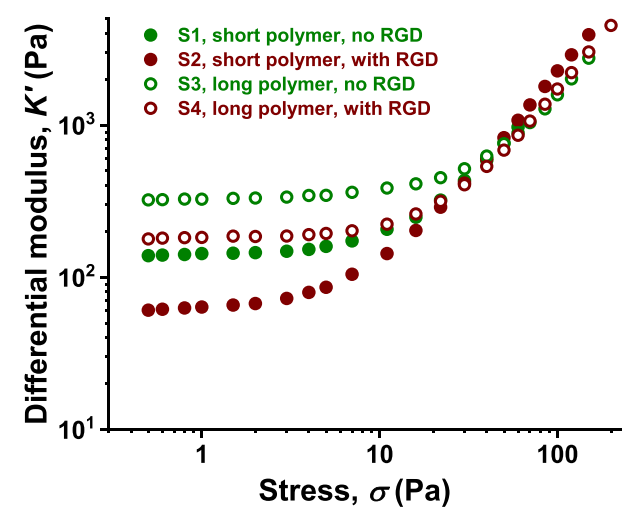

Figure 1. Synthetic fibrous hydrogels used in this study. (a) 4 polymer gels (S1-S4) are used, namely a combination between short/long polymers and decoration with/without RGD peptides. (b) Thermosensitive behavior of PIC gels. The solution state and the gel state of PIC are reversible by changing the temperature. (c) Structure of the PIC-GRGDS with a DBCO-PEG4 linker. Note that $\sim 1 \%$ of all monomers is functionalized with the RGD peptide. (d) Storage modulus $G^{\prime}$ as a function of temperature. Gels form approximately at $15{ }^{\circ} \mathrm{C}$. (e) Differential modulus $K^{\prime}$ as a function of applied stress. The polymer concentration is fixed at $1 \mathrm{mg} / \mathrm{mL}$ and freshly dissolved in $\alpha$-MEM cell culture medium.

architecture and (nonlinear) elasticity which is common for biogels of structural biopolymers, such as collagen and fibrin, that (partially) comprise the natural extracellular matrix. ${ }^{19}$ This property is often termed "strain-stiffening", and the sensitivity and responsiveness to external stress can be tuned by changing various parameters, ${ }^{20,21}$ including the polymer length. ${ }^{22}$ For PIC gels, the molecular weights are readily varied and, additionally, biologically relevant moieties such as cell-adhesive peptides, including the well-known Gly-Arg-Gly-Asp-Ser (GRGDS), are introduced straightforwardly via a copper-free strain-promoted azide-alkyne cycloaddition (SPAAC). ${ }^{23}$ The RGD motif enables cells to adhere to the PIC hydrogels by integrin-mediated binding. This simple and minimal PIC-based system provides the possibility to modulate cell adhesion capacity, cell motility, proliferation, vascularization, and cytocompatibility, which can be manipulated to produce different micromilieus. ${ }^{24,25}$ Moreover, the cells in the PIC hydrogel network can generate internal stresses resulting in the ability to change the local mechanical microenvironment. ${ }^{22}$ 

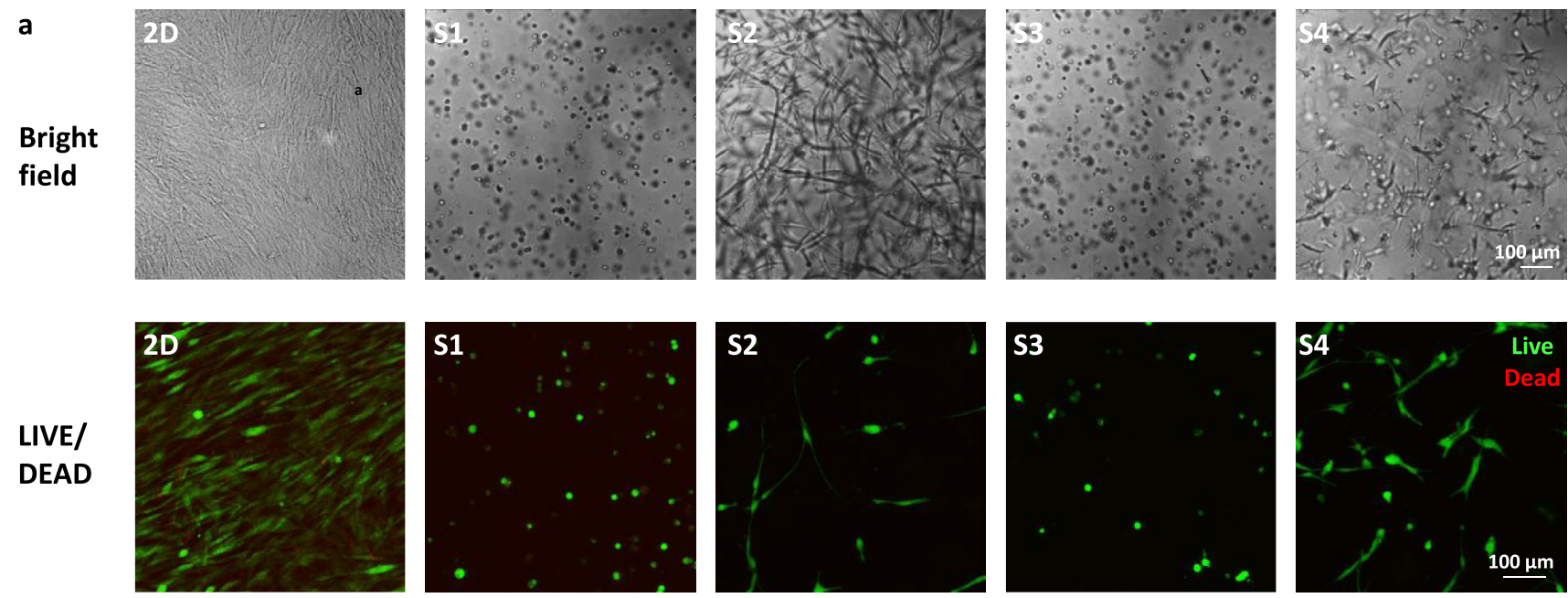

b

WST-1

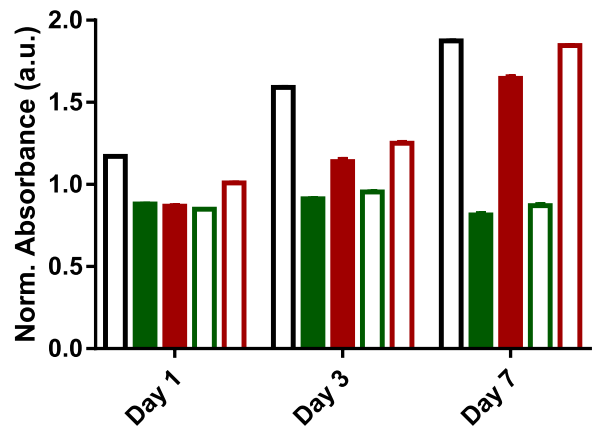

Picogreen

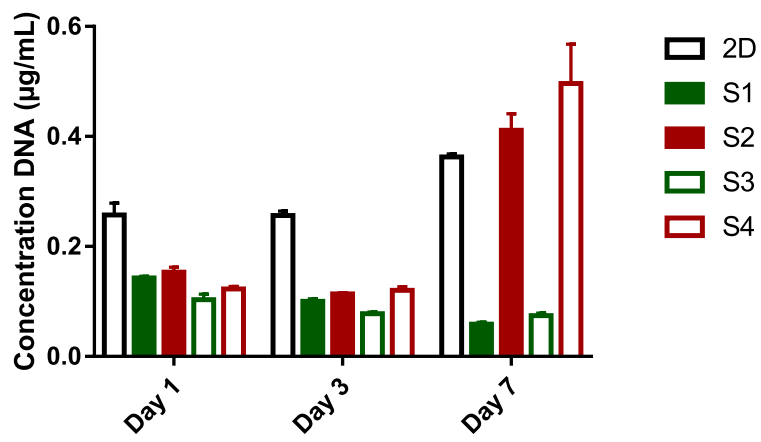

Figure 2. Stem cell behavior is influenced by matrix properties. (a) Representative bright field images (top) and z-stacks of LIVE/DEAD imaging (bottom) show morphology and viability of stem cells at day 7 (bright field) and day 8 (LIVE/DEAD) in all conditions, respectively. Stem cells show the most pronounced spreading in gels functionalized with cell adhesive peptides and with shorter polymers (S2). (b) WST-1 assay and PicoGreen DNA quantification assay show metabolic activities and proliferation of cells in all conditions, respectively. A similar trend in both assays confirms that cell-adhesive peptides promote cell proliferation in $3 \mathrm{D}$ culture.

In this work, we take advantage of the customizable nature of PIC gels, which provides the possibility to tune the cellular 3D microenvironment and ultimately produce different stem cell secretomes. We studied four different gels (stiff-soft, RGD-no RGD) to culture hASCs and found clear differences in the secretomes by immunoassays. We then exploited the different secretomes and applied these $\mathrm{CM}$ in functional wound healing assays. Importantly, the rate of wound closure depends on the CM applied, showcasing the advantages of the 3D synthetic matrices in tuning stem cell secretome.

\section{RESULTS AND DISCUSSION}

2.1. Synthesis and Functionalization of Polymers. For this study, we prepared four different culture matrices based on polymers where we vary the polymer contour length $L_{C}$ and presence of cell-adhesive peptides. To obtain materials of different contour lengths, we carried out PIC polymerizations with varying monomer to catalyst ratios, ${ }^{22}$ yielding polymer $\mathbf{P} \mathbf{1}$ and P2 with $L_{\mathrm{C}}=160$ and $230 \mathrm{~nm}$, respectively (Table 1 ). Note that in the polymerization $3.3 \%$ azide, $\left(\mathrm{N}_{3}\right)$-appended monomer was copolymerized for postmodification. The celladhesive RGD peptide was equipped with a DBCO-terminated PEG spacer and conjugated to P1 and P2 through the highly efficient SPAAC reaction; ${ }^{22}$ such that, on average, $1 \%$ of the monomers in the polymer chain carries a peptide, which corresponds to an average theoretical spacing of $10 \mathrm{~nm}$ between adjacent peptides along the polymer chain (Figure $1 \mathrm{a}, \mathrm{c})$. Details are given in the Supporting Information.

To prepare hydrogels, polymers were dissolved in $\alpha$-MEM (concentration $1 \mathrm{mg} / \mathrm{mL}$ ) at low temperatures $\left(5{ }^{\circ} \mathrm{C}\right.$ ) and subsequently heated to $37^{\circ} \mathrm{C}$ where the gel is formed instantly. The mechanical properties of the gels were measured using oscillatory shear rheology. The rheology experiments clearly show that all polymer gels possess a lower critical solution temperature or gelation temperature of approximately $15{ }^{\circ} \mathrm{C}$ (Figure $1 \mathrm{~b}, \mathrm{~d}$ ). In the linear viscoelastic (LVE) regime, all gels are considered relatively soft with a stiffness or storage modulus $G^{\prime}$ between 50 and $350 \mathrm{~Pa}$. Comparison between different gels shows that the gels that are prepared from polymers with a reduced polymer length are softer, which is in line with the previous results. ${ }^{22}$ Besides, the introduction of low amounts of RGD also reduces the stiffness of the gel (Table 1).

In a prestress protocol, we measured the mechanical properties of the hydrogels under stress and find the critical stress $\sigma_{\mathrm{c}}$ where the mechanical response changes from the LVE to the stiffening regime. The results are given as the differential modulus $K^{\prime} \equiv \partial \sigma / \partial \gamma$, which more accurately describes the material's stiffness at a specific stress (Figure 1e). The softer gels have lower critical stresses; that is, at much lower applied stress, they will transfer into a mechanical stiffening regime. Consequently, at higher stresses, the stiffness of all gels 
a

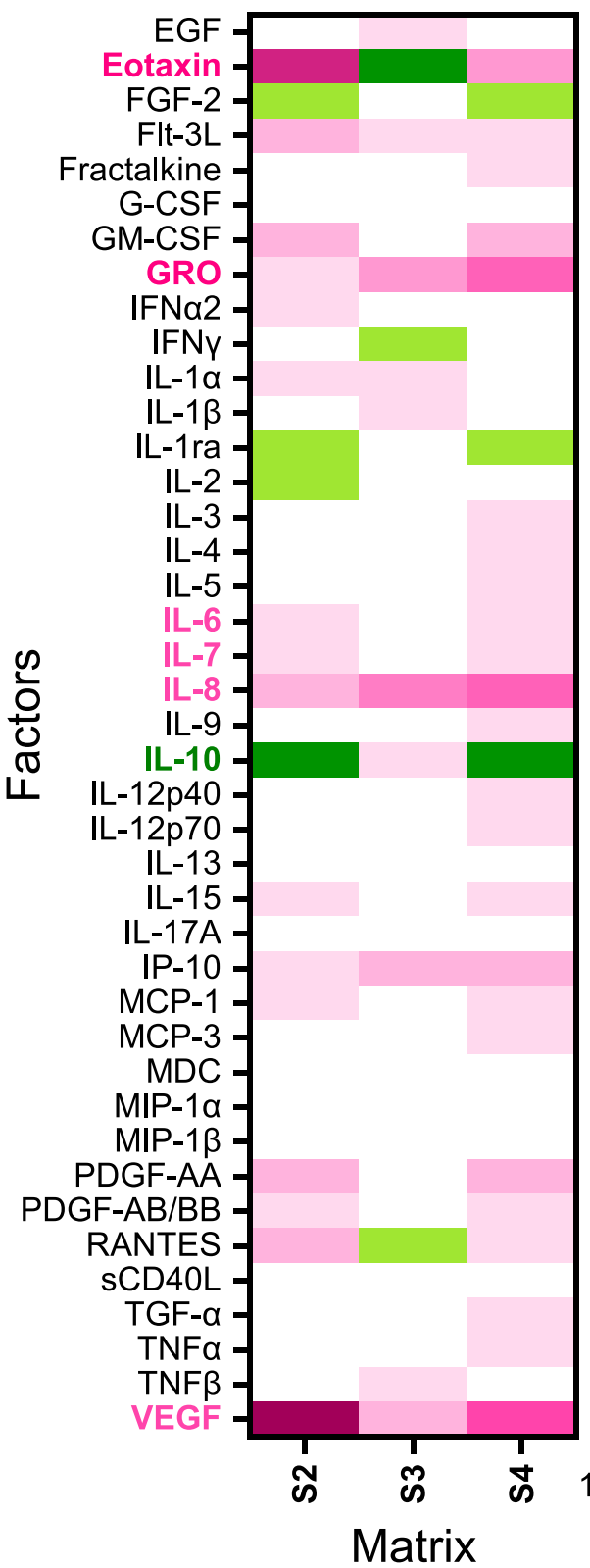

b

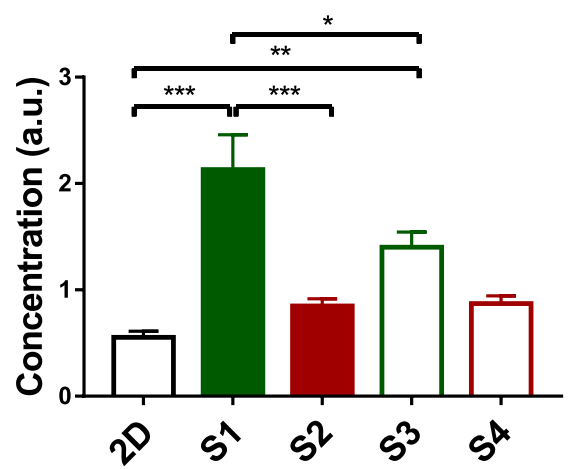

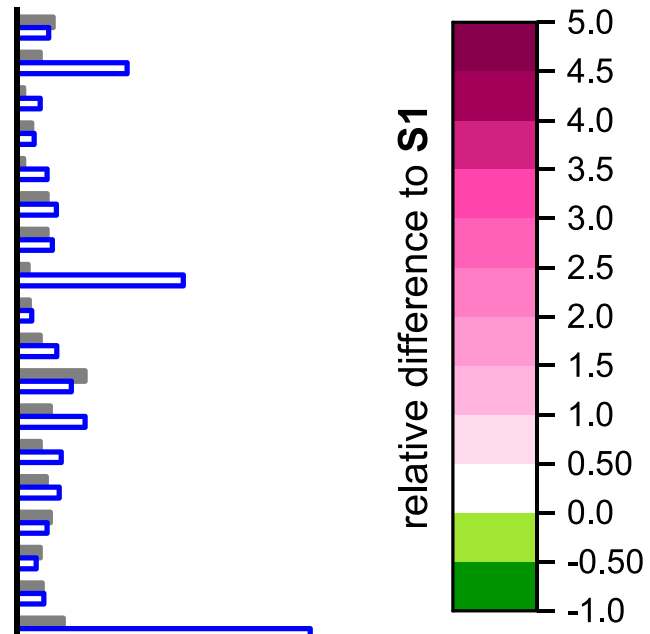

absolute concentrations

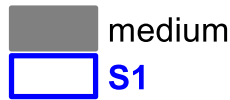

Figure 3. Stem cell secretome is influenced by matrix properties. (a) Quantification of the stem cell secretome by the multiplex assay. The heat map (left) shows fold increase in cytokine expression of S2, S3, and S4 in comparison to S1. Cytokines with marked higher expressions in RGDappended matrices are labeled in pink; IL-10, which is higher expressed in inert matrices, is labeled in dark green. The bar chart (right) shows the absolute concentrations of cytokines in $\mathbf{S 1}$ and the medium control. See Figure S2 for the absolute expression levels. Cell density: 500,000 cells/ $\mathrm{mL}$; conditioned medium was refreshed every 2 days, and the medium at day 6 was collected for the measurement. (b) ELISA reveals the average concentration of IL-10 in CM (left) and normalized per cell (right) for all samples. Cell density: 200,000 cells/mL; conditioned medium was refreshed on day 1,3 , and 7 ; the medium at day 7 was collected for the measurement. 
converges, and at relatively low stress of $30 \mathrm{~Pa}$, all materials display uniform mechanics, as clear from the overlapping $K^{\prime}$ data.

2.2. Morphology, Metabolism, and Proliferation of Stem Cells are Regulated by Gel Properties. hASCs were encapsulated and cultured in four different gels as well as on tissue culture plastic as a $2 \mathrm{D}$ control. Within the 7 day culture, the stem cells adapted an elongated morphology in RGDappended gels S2 and S4, with a more pronounced spreading in short-polymer gel S2. In contrast, the cells in the non-RGDmodified materials displayed no significant protrusion, which is consistent with our previous findings in gels with higher polymer concentrations (Figure $2 \mathrm{a}$ top row). ${ }^{22}$ LIVE/DEAD imaging confirms the good viability of stem cells in all conditions (Figure 2a bottom row). The WST-1 proliferation assay reveals that stem cells show an increased metabolic activity throughout the 7 day culture in RGD gels and on 2D surfaces, whereas in the "inert" gels, the metabolic activity remains constant, suggesting the absence of proliferation (Figure $2 \mathrm{~b}$ left panel). The PicoGreen DNA quantification assay, in line with the WST-1 results, reveals that the amount of total DNA increases from day 3 onward in the RGDcontaining PIC gels and on 2D surfaces but does not show any increase (even a small decrease) in the inert gels (Figure $2 \mathrm{~b}$ right panel). Together, the data suggest that human stem cells require sufficient cell-matrix adhesion in their 3D microenvironments to display a proliferative phenotype.

2.3. Culture Dimensionality and Cell Adhesion Sites Influence the Secretome of Stem Cells. The major differences between morphologies and metabolisms of hASCs in the 4 cultures, particularly between adherent and nonadherent matrices, prompted us to screen the secretomes. In a first approach, we performed a multiplex assay (panel of 41 bioactive factors) on all 3D samples (S1, S2, S3, and S4) to study whether the observed differences translate into an altered paracrine content in the corresponding CM. The expression levels of different molecules are summarized in Figure 3. We present the absolute concentrations in $\mathbf{S 1}$ and the control medium in a bar chart and the relative difference of $\mathbf{S 2 - S 4}$ to $\mathbf{S 1}$ in a heat map (Figure $3 \mathrm{a}$ and Table S1 for all absolute values). Among the 41 molecules in the panel, the concentrations of eotaxin, GRO, IL-6, IL-7, IL-8, IL-10, MCP-1, and vascular endothelial growth factor (VEGF) in CM from $3 \mathrm{D}$ cultures are several orders of magnitude higher than the control, suggesting an active production of these cytokines by stem cells in response to their PIC surrounding. While most of these proteins are expressed more in the RGD-functionalized gels (S2, S4) than in inert matrices $(\mathbf{S 1}, \mathbf{S 3})$ or at least show similar expressions, IL-10 is the only exception that is upregulated in the inert matrices. To acquire a comprehensive understanding of IL-10 expression, we performed ELISA on all samples (Figures $3 \mathrm{~b}$ and S2). Indeed, we detected higher expressions of IL-10 from all 3D conditions compared with the $2 \mathrm{D}$ culture and the highest concentrations in inert gels $\mathbf{S} \mathbf{1}$ and S3. The effect is even stronger when the IL-10 expression is normalized to the number of cells considering the reduced proliferation in these conditions (Figure $3 \mathrm{~b}$ ).

IL-10 is a pleiotropic cytokine, involved in immune modulation as it has anti-inflammatory properties and prevents autoimmunity. ${ }^{26-28}$ Moreover, in vivo studies have shown that IL-10 is involved in enhanced and scarless wound healing. This observation, however, was mainly achieved by IL-10-mediated immune cells modulation. ${ }^{29-31}$ Meanwhile, earlier studies on stem cell-expressed IL-10 mainly used genetic modifications to overexpress the cytokine. ${ }^{32,33}$ We note that Stucky et al. encapsulated mesenchymal stem cells (MSCs) in alginate microspheres but did not observe a significant change in IL-10 expression in comparison with a monolayer culture. ${ }^{34}$ Our results show that IL-10 expression of MSCs can be effectively upregulated using a (nonadherent) 3D matrix. The (small) increase in expression of IL-10 for gels prepared from a short polymer gel (S1) compared to S3 disappears after normalization to the cell number.

In the absence of cell adhesion peptides, the mechanical confinement by the ECM has been reported to dominate cytokine expression from cells. Along the same lines, Lee et al. has reported an altered secretion of IL- $1 \beta$ by chondrocytes in inert alginate gels with different viscoelastic properties that translated to various degrees of cell volume restriction in the 3D microenvironment. ${ }^{35}$ Jain and Vogel suggested that cytokine-associated biophysical pathways in macrophages impacted by spatial confinement include lipopolysaccharide (LPS)-activated transcriptional programs and nuclear translocation of MRTF-A. ${ }^{36}$ In the context of our study, we speculate that cell volume restriction, inhibition of actin polymerization, a lack of cell-matrix, and cell-cell interactions all contribute to the activation of IL-10 production via already known or not yet discovered biophysical signaling pathways. We note that currently, the conditions for IL-10 production by mesenchymal stem cells are debated. Primarily, the uncertainty is the result of a lacking consistency in experimental conditions. ${ }^{37}$ Synthetic ECM models such as PIC are able to better control the experimental variables.

Interestingly, we did not observe any significant difference between short and long polymer gels functionalized with RGD peptides despite the clear difference in the stem cell morphology. There is a general consensus that the nonlinear mechanics of fibrous ECMs enable the embedded contractile cells to actively modulate the stiffness of microtissues, via actin-myosin contractility. ${ }^{38,39}$ In light of this, we consider that the stem cells are able to actively stiffen the matrix to a desired value, regardless of the initial stiffness of PIC-RGD gels. Consequently, the difference in mechanical properties between the short and long polymer gels is minimized after the cellular intervention. In macroscale shear rheology, an overlap of gel stiffness is seen for all gels already at modest (external) stresses. We propose that the encapsulated cells in gels similarly exert (internal) stresses that can drive gel mechanics into a similar level. Moreover, cell-cell contact can be observed in both the $2 \mathrm{D}$ culture and $3 \mathrm{D}$ cultures with RGD, where IL-10 expressions are both low, which supports the aforementioned hypothesis.

Another signaling protein from the panel, VEGF, also raised our interest because of its crucial role in promoting vascularization. ELISA was performed on the VEGF, and we observed a higher expression in $2 \mathrm{D}$ culture than all $3 \mathrm{D}$ cultures (Figure S2). We hypothesize that a plethora of stimuli, including culture dimensionality, substrate stiffness, and cellcell interactions, influence the secretion of paracrine molecules, and each different molecule has its own favored combination of stimuli. Because of the key role of IL-10 in wound healing and large expression differences, we continued our study on IL10.

2.4. CM from 3D Inert Gels Promote Wound Healing of Fibroblasts and IL-10 Plays a Role. As an example to demonstrate the functional effects of the collected secretomes, 
a
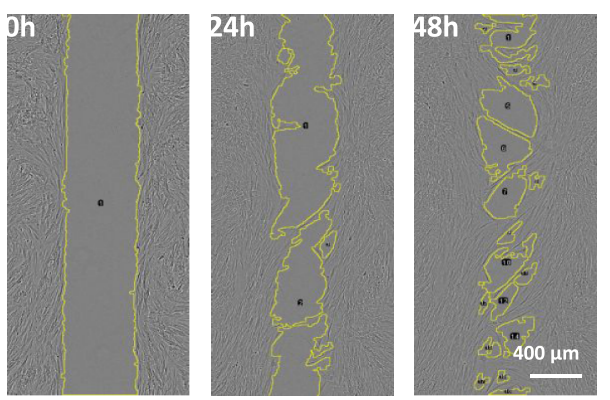

C

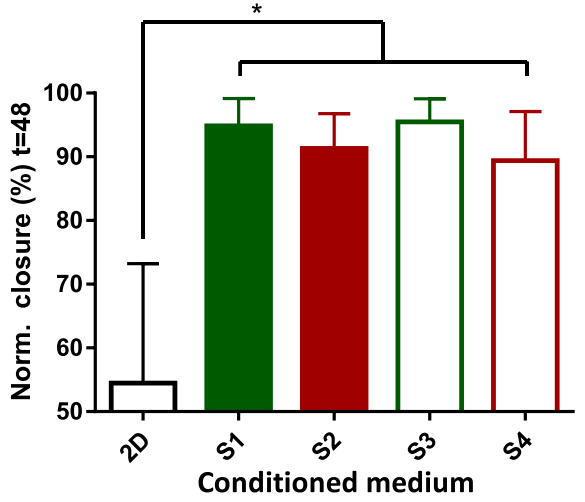

e
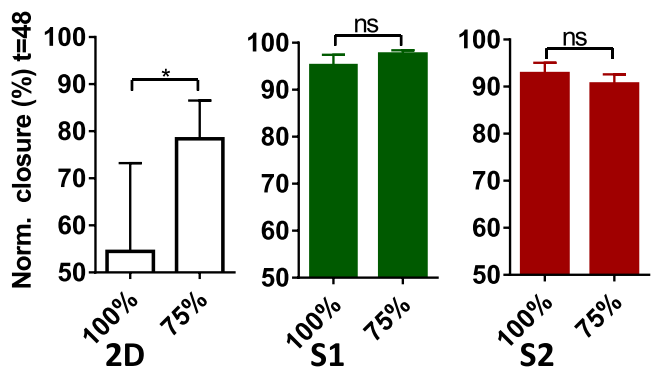

b

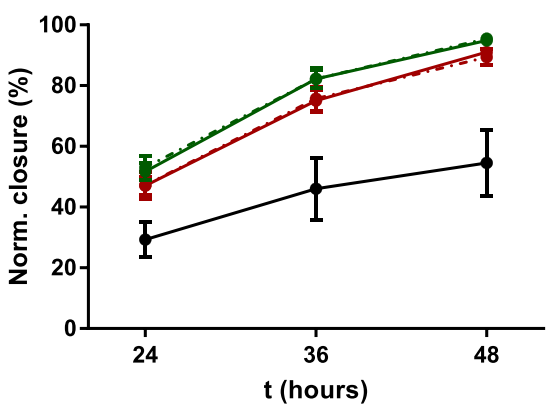

- 2D

- S1

- S2

-.. $\mathrm{s} 3$

... 54

d
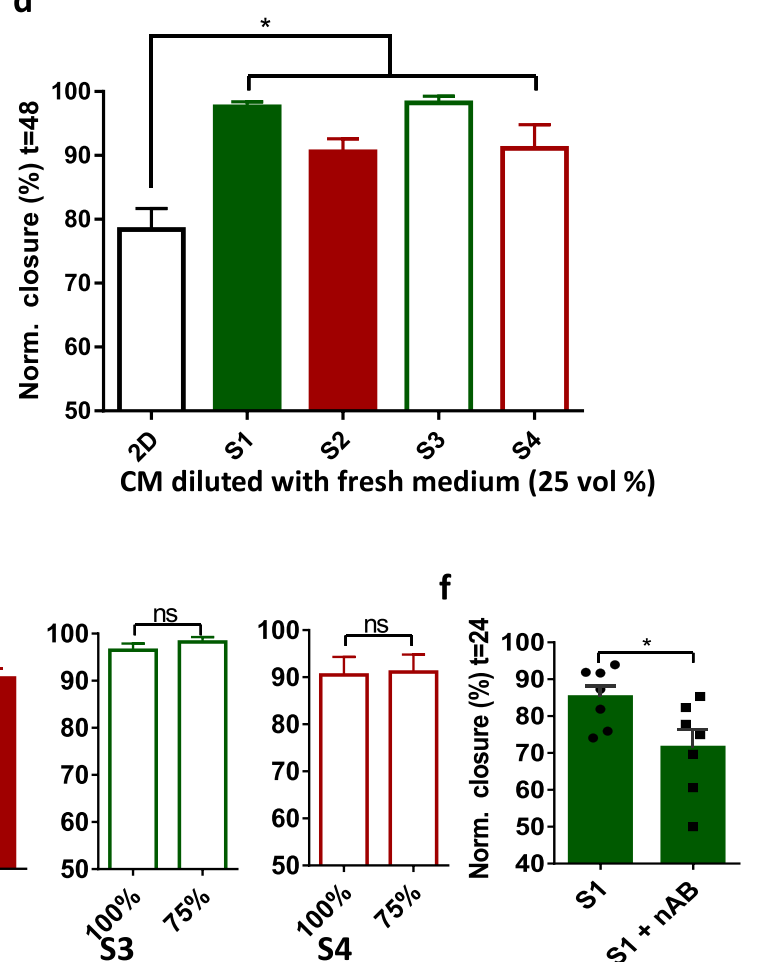

Figure 4. Fibroblast wound healing assays. (a) Representative bright field images show a wound closure with time. The area of wound is analyzed by ImageJ. (b) Wound closure in undiluted CM with time in all conditions. (c,d) Wound closure in undiluted (c) and diluted (d) CMs after $48 \mathrm{~h}$. (e) Comparison between undiluted and diluted CMs in all conditions. (f) Comparison between wound closure in CMs S1 and S1 + IL-10 nAB after $24 \mathrm{~h}$. For comparisons between condition variants, a one-way ANOVA with a Tukey post hoc test for pairwise comparisons was used (*P $\leq$ $0.05)$. For comparisons between $S 1$ and $S 1+\mathrm{IL}-10 \mathrm{nAB}$, a two-tailed, student-t-test was used $\left({ }^{*} P \leq 0.05\right)$. Note that we used a volume of $400 \mu \mathrm{L}$ for the $\mathrm{CM}$ in the $2 \mathrm{D}$ condition. $n=3$ independent experiments.

we applied the CM in a fibroblast wound healing assay. Briefly, two islands of fibroblasts were cultured on tissue culture plastic separated by a well-defined barrier $(500 \mu \mathrm{m})$. Then, the barrier was removed and the medium was replaced by CM from the stem cell experiments. The closure of the artificial wound was then monitored for $48 \mathrm{~h}$ (Figure $4 \mathrm{a}$ ). The results reveal that $\mathrm{CM}$ from all 3D stem cell cultures induce a much faster and more complete wound closure than $\mathrm{CM}$ from the $2 \mathrm{D}$ culture. Statistically, the CM from the 3D stem cell cultures in RGDfree conditions (with increased IL-10 levels) show a similar wound closure rate compared with CM from cells in matrices with RGD (Figure 4b,c). The results suggest that culture dimensionality (2D vs 3D) dominates over the influence of paracrine effects or nonlinear mechanics of the gels. See Figure S3 for an overview of bright field images used for wound closure quantification.

To confirm that the observed differences in the wound closure rate are not simply due to nutrient depletion, we supplemented the CM with fresh $\alpha$-MEM medium (3:1) and repeated the assay. For 3D cultures, we observe no significant differences, suggesting that nutrient depletion does not play a role. Only for the $\mathrm{CM}$ of the $2 \mathrm{D}$ control, we observe that the wound closure rate increases for the $\alpha$-MEM-supplemented CM. After $48 \mathrm{~h}$, however, wound closure is still slower than in any CM of the 3D hASC cultures (Figure 4d,e). A WST-1 proliferation assay was performed on fibroblasts incubated in $\mathrm{CM}$ from all culture conditions after $48 \mathrm{~h}$, and no statistical difference could be observed among 3D conditions (Figure S4). All these data confirm that the difference in wound closure between samples is most likely caused by components in the $\mathrm{CM}$ and not by the nutrient consumption.

To validate the role of IL-10, we employed an IL-10 neutralizing antibody $(\mathrm{nAB})$ in the $\mathrm{CM}$ from the RGD-free gel S1. Results suggest that, indeed, the $n A B$ retards wound healing (Figure 4f). Interestingly, we did not observe significantly faster wound healing in $\alpha$-MEM media (with $1 \%$ 
FSC) supplemented with IL-10 compared with control culture media (Figure S5). In the field of regenerative medicine, the overexpression of IL-10 has been proved highly beneficial in scarless wound healing by regulating the fibroblast function and ECM remodeling. ${ }^{30}$ Our findings once again confirm the key role of IL-10 in tissue regeneration but also strongly indicate that IL-10 works in concert with other factors present in the CM. We hypothesize that fibroblasts need specific biochemical cues to accelerate wound healing and that these cues are absent in normal culture medium. Future research needs to investigate which factor is key to the process.

\section{CONCLUSIONS}

In this study, we demonstrate that the secretome of stem cells is readily tuned by their (3D) culture conditions. In the example of hASC-laden PIC gels that lack cell-adhesive peptides, we observe an increased IL-10 production, which is involved in wound healing and has a therapeutic potential.

Zooming out beyond the current study where we focused on simple cell-gel interactions and immunological responses, we believe that this work marks the advent of new approaches to generate customized CM. The application of well-defined, synthetic matrices is crucial. They offer the opportunity for highly controlled manipulation of the network architecture and physical and mechanical properties of the hydrogel. Maybe, more importantly, they allow for the introduction of bioactive factors to stimulate stem cells, beyond the simple RGD cellbinding peptide that we varied in this example. As such, synthetic matrices, such as the PIC gel, will allow virtually endless variations of structures and properties to ultimately generate an optimized conditioned medium.

\section{ASSOCIATED CONTENT}

\section{(3) Supporting Information}

The Supporting Information is available free of charge at https://pubs.acs.org/doi/10.1021/acsami.0c16208.

Experimental section and supplementary figures (PDF)

\section{AUTHOR INFORMATION}

\section{Corresponding Authors}

Silvia M. Mihaila - Utrecht Institute for Pharmaceutical Sciences, Division of Pharmacology, Utrecht University, 3854 CG Utrecht, The Netherlands; Email: s.mihaila@uu.nl

Paul H. J. Kouwer - Institute for Molecules and Materials, Radboud University, 6525 AJ Nijmegen, The Netherlands; ○ orcid.org/0000-0002-2760-191X; Email: p.kouwer@ science.ru.nl

\section{Authors}

Kaizheng Liu - Institute for Molecules and Materials, Radboud University, 6525 AJ Nijmegen, The Netherlands; (ㄱ) orcid.org/0000-0001-8555-4536

Tomas Veenendaal - Institute for Molecules and Materials, Radboud University, 6525 AJ Nijmegen, The Netherlands

Maury Wiendels - Institute for Molecules and Materials, Radboud University, 6525 AJ Nijmegen, The Netherlands

Alejandra M. Ruiz-Zapata - Radboud Institute for Molecular Life Sciences, Department of Obstetrics and Gynecology, Radboud University Medical Center, 6500 HB Nijmegen, The Netherlands

Justin van Laar - Institute for Molecules and Materials, Radboud University, 6525 AJ Nijmegen, The Netherlands
Rafail Kyranas - Institute for Molecules and Materials, Radboud University, 6525 AJ Nijmegen, The Netherlands

Hilde Enting - Institute for Molecules and Materials, Radboud University, 6525 AJ Nijmegen, The Netherlands

Bram van Cranenbroek - Lab Medical Immunology, Laboratory Medicine, Radboud University Medical Centre, 6500 HB Nijmegen, The Netherlands

Hans J. P. M. Koenen - Lab Medical Immunology, Laboratory Medicine, Radboud University Medical Centre, 6500 HB Nijmegen, The Netherlands

Egbert Oosterwijk - Radboud Institute for Molecular Life Sciences, Department of Urology, Radboud University Medical Centre, 6500 HB Nijmegen, The Netherlands

Complete contact information is available at: https://pubs.acs.org/10.1021/acsami.0c16208

\section{Author Contributions}

K.L. worked on polymer synthesis, rheology, cell culture, and encapsulation, imaging and project management. T.V. and M.W. worked on wound healing assays and data analysis. A.M.R.-Z. worked on fibroblast culture and manuscript editing. J.v.L., R.K., and H.E. worked on encapsulation, CM collection and proliferation assays. S.M., E.O., and P.H.J.K. supervised the experiments. The manuscript was written through contributions of all authors. All authors have given approval to the final version of the manuscript.

\section{Notes}

The authors declare no competing financial interest.

\section{ACKNOWLEDGMENTS}

We thank Coen van Schayk for the support on polymer synthesis, Dorien Timmessen and Kees Jansen for their support on cell studies, Paul Rijken for the support on image analysis, Roel Hammink for the suggestions on antibody blocking experiments, Melissa van Velthoven and Jyoti Kumari for the support on fluorescence imaging, and Guillermo Solis for the support on proof reading. This project has received funding from the European Union's Horizon 2020 research and innovation programme under the Marie Skłodowska-Curie grant agreements no. 642687 (K.L.).

\section{REFERENCES}

(1) Daneshmandi, L.; Shah, S.; Jafari, T.; Bhattacharjee, M.; Momah, D.; Saveh-Shemshaki, N.; Lo, K. W.; Laurencin, C. T. Emergence of the Stem Cell Secretome in Regenerative Engineering. Trends Biotechnol. 2020, 38, 1373.

(2) Hocking, A. M.; Gibran, N. S. Mesenchymal Stem Cells: Paracrine Signaling and Differentiation During Cutaneous Wound Repair. Exp. Cell Res. 2010, 316, 2213-2219.

(3) Zhang, W.; Bai, X.; Zhao, B.; Li, Y.; Zhang, Y.; Li, Z.; Wang, X.; Luo, L.; Han, F.; Zhang, J.; Han, S.; Cai, W.; Su, L.; Tao, K.; Shi, J.; $\mathrm{Hu}, \mathrm{D}$. Cell-Free Therapy Based on Adipose Tissue Stem CellDerived Exosomes Promotes Wound Healing Via the Pi3k/Akt Signaling Pathway. Exp. Cell Res. 2018, 370, 333-342.

(4) Fu, Y.; Karbaat, L.; Wu, L.; Leijten, J.; Both, S. K.; Karperien, M. Trophic Effects of Mesenchymal Stem Cells in Tissue Regeneration. Tissue Eng., Part B 2017, 23, 515-528.

(5) L, P. K.; Kandoi, S.; Misra, R.; S, V.; K, R.; Verma, R. S. The Mesenchymal Stem Cell Secretome: A New Paradigm Towards CellFree Therapeutic Mode in Regenerative Medicine. Cytokine Growth Factor Rev. 2019, 46, 1-9.

(6) Salgado, A. J.; Gimble, J. M.; Costa, B. M. The Cell Secretome in Personalized and Regenerative Medicine. Biochimie 2018, 155, 1. 
(7) Rad, F.; Ghorbani, M.; Mohammadi Roushandeh, A.; Habibi Roudkenar, M. Mesenchymal Stem Cell-Based Therapy for Autoimmune Diseases: Emerging Roles of Extracellular Vesicles. Mol. Biol. Rep. 2019, 46, 1533-1549.

(8) Baglio, S. R.; Pegtel, D. M.; Baldini, N. Mesenchymal Stem Cell Secreted Vesicles Provide Novel Opportunities in (Stem) Cell-Free Therapy. Front. Physiol. 2012, 3, 359.

(9) Ribeiro, C. A.; Fraga, J. S.; Graos, M.; Neves, N. M.; Reis, R. L.; Gimble, J. M.; Sousa, N.; Salgado, A. J. The Secretome of Stem Cells Isolated from the Adipose Tissue and Wharton Jelly Acts Differently on Central Nervous System Derived Cell Populations. Stem Cell Res. Ther. 2012, 3, 18.

(10) Redondo-Castro, E.; Cunningham, C.; Miller, J.; Martuscelli, L.; Aoulad-Ali, S.; Rothwell, N. J.; Kielty, C. M.; Allan, S. M.; Pinteaux, E. Interleukin-1 Primes Human Mesenchymal Stem Cells Towards an Anti-Inflammatory and Pro-Trophic Phenotype in Vitro. Stem Cell Res. Ther. 2017, 8, 79.

(11) Silva, N. A.; Moreira, J.; Ribeiro-Samy, S.; Gomes, E. D.; Tam, R. Y.; Shoichet, M. S.; Reis, R. L.; Sousa, N.; Salgado, A. J. Modulation of Bone Marrow Mesenchymal Stem Cell Secretome by Ecm-Like Hydrogels. Biochimie 2013, 95, 2314-2319.

(12) Redondo-Castro, E.; Cunningham, C. J.; Miller, J.; Brown, H.; Allan, S. M.; Pinteaux, E. Changes in the Secretome of TriDimensional Spheroid-Cultured Human Mesenchymal Stem Cells in Vitro by Interleukin-1 Priming. Stem Cell Res. Ther. 2018, 9, 11.

(13) Edmondson, R.; Broglie, J. J.; Adcock, A. F.; Yang, L. ThreeDimensional Cell Culture Systems and Their Applications in Drug Discovery and Cell-Based Biosensors. Assay Drug Dev. Technol. 2014, 12, 207-218.

(14) Baker, B. M.; Chen, C. S. Deconstructing the Third Dimension - How 3d Culture Microenvironments Alter Cellular Cues. J. Cell Sci. 2012, 125, 3015-3024.

(15) Xu, Y.; Shi, T.; Xu, A.; Zhang, L. 3d Spheroid Culture Enhances Survival and Therapeutic Capacities of Mscs Injected into Ischemic Kidney. J. Cell. Mol. Med. 2016, 20, 1203-1213.

(16) Murphy, K. C.; Whitehead, J.; Zhou, D.; Ho, S. S.; Leach, J. K. Engineering Fibrin Hydrogels to Promote the Wound Healing Potential of Mesenchymal Stem Cell Spheroids. Acta Biomater. 2017, 64, 176-186.

(17) Carter, K.; Lee, H. J.; Na, K.-S.; Fernandes-Cunha, G. M.; Blanco, I. J.; Djalilian, A.; Myung, D. Characterizing the Impact of $2 \mathrm{~d}$ and $3 \mathrm{~d}$ Culture Conditions on the Therapeutic Effects of Human Mesenchymal Stem Cell Secretome on Corneal Wound Healing in Vitro and Ex Vivo. Acta Biomater. 2019, 99, 247-257.

(18) Lutolf, M. P.; Gilbert, P. M.; Blau, H. M. Designing Materials to Direct Stem-Cell Fate. Nature 2009, 462, 433-441.

(19) Kouwer, P. H. J.; Koepf, M.; Le Sage, V. A. A.; Jaspers, M.; van Buul, A. M.; Eksteen-Akeroyd, Z. H.; Woltinge, T.; Schwartz, E.; Kitto, H. J.; Hoogenboom, R.; Picken, S. J.; Nolte, R. J. M.; Mendes, E.; Rowan, A. E. Responsive Biomimetic Networks from Polyisocyanopeptide Hydrogels. Nature 2013, 493, 651-655.

(20) Jaspers, M.; Dennison, M.; Mabesoone, M. F. J.; MacKintosh, F. C.; Rowan, A. E.; Kouwer, P. H. J. Ultra-Responsive Soft Matter from Strain-Stiffening Hydrogels. Nat. Commun. 2014, 5, 5808.

(21) Jaspers, M.; Rowan, A. E.; Kouwer, P. H. J. Tuning Hydrogel Mechanics Using the Hofmeister Effect. Adv. Funct. Mater. 2015, 25, 6503-6510.

(22) Liu, K.; Mihaila, S. M.; Rowan, A.; Oosterwijk, E.; Kouwer, P. H. J. Synthetic Extracellular Matrices with Nonlinear Elasticity Regulate Cellular Organization. Biomacromolecules 2019, 20, 826834.

(23) Mandal, S.; Eksteen-Akeroyd, Z. H.; Jacobs, M. J.; Hammink, R.; Koepf, M.; Lambeck, A. J. A.; van Hest, J. C. M.; Wilson, C. J.; Blank, K.; Figdor, C. G.; Rowan, A. E. Therapeutic Nanoworms: Towards Novel Synthetic Dendritic Cells for Immunotherapy. Chem. Sci. 2013, 4, 4168-4174.

(24) Ruoslahti, E. Rgd and Other Recognition Sequences for Integrins. Annu. Rev. Cell Dev. Biol. 1996, 12, 697-715.
(25) Ruoslahti, E.; Pierschbacher, M. D. Arg-Gly-Asp - a Versatile Cell Recognition Signal. Cell 1986, 44, 517-518.

(26) Mollazadeh, H.; Cicero, A. F. G.; Blesso, C. N.; Pirro, M.; Majeed, M.; Sahebkar, A. Immune Modulation by Curcumin: The Role of Interleukin-10. Crit. Rev. Food Sci. Nutr. 2019, 59, 89-101.

(27) Fillatreau, S.; Sweenie, C. H.; McGeachy, M. J.; Gray, D.; Anderton, S. M. B Cells Regulate Autoimmunity by Provision of Il-10. Nat. Immunol. 2002, 3, 944-950.

(28) Sanjabi, S.; Zenewicz, L. A.; Kamanaka, M.; Flavell, R. A. AntiInflammatory and Pro-Inflammatory Roles of Tgf-Beta, Il-10, and Il22 in Immunity and Autoimmunity. Curr. Opin. Pharmacol. 2009, 9, 447-453.

(29) Peranteau, W. H.; Zhang, L.; Muvarak, N.; Badillo, A. T.; Radu, A.; Zoltick, P. W.; Liechty, K. W. Il-10 Overexpression Decreases Inflammatory Mediators and Promotes Regenerative Healing in an Adult Model of Scar Formation. J. Invest. Dermatol. 2008, 128, 18521860.

(30) King, A.; Balaji, S.; Le, L. D.; Crombleholme, T. M.; Keswani, S. G. Regenerative Wound Healing: The Role of Interleukin-10. $A d v$. Wound Care 2014, 3, 315-323.

(31) Liechty, K. W.; Kim, H. B.; Adzick, N. S.; Crombleholme, T. M. Fetal Wound Repair Results in Scar Formation in Interleukin-10Deficient Mice in a Syngeneic Murine Model of Scarless Fetal Wound Repair. J. Pediatr. Surg. 2000, 35, 866-873.

(32) Nakajima, M.; Nito, C.; Sowa, K.; Suda, S.; Nishiyama, Y.; Nakamura-Takahashi, A.; Nitahara-Kasahara, Y.; Imagawa, K.; Hirato, T.; Ueda, M.; Kimura, K.; Okada, T. Mesenchymal Stem Cells Overexpressing Interleukin-10 Promote Neuroprotection in Experimental Acute Ischemic Stroke. Mol. Ther.-Methods Clin. Dev. 2017, 6, 102-111.

(33) Xiao, S.; Huang, G. T.; Wei, Z. R.; Nie, K. Y.; Liu, Z. Y.; Deng, C. L.; Wang, D. L. Il-10 Gene-Modified Human Amniotic Mesenchymal Stem Cells Augment Regenerative Wound Healing by Multiple Synergistic Effects. Stem Cell. Int. 2019, 2019, 9158016.

(34) Stucky, E. C.; Schloss, R. S.; Yarmush, M. L.; Shreiber, D. I. Alginate Micro-Encapsulation of Mesenchymal Stromal Cells Enhances Modulation of the Neuro-Inflammatory Response. Cytotherapy 2015, 17, 1353-1364.

(35) Lee, H.-p.; Gu, L.; Mooney, D. J.; Levenston, M. E.; Chaudhuri, O. Mechanical Confinement Regulates Cartilage Matrix Formation by Chondrocytes. Nat. Mater. 2017, 16, 1243-1251.

(36) Jain, N.; Vogel, V. Spatial Confinement Downsizes the Inflammatory Response of Macrophages. Nat. Mater. 2018, 17, 1134.

(37) Kyurkchiev, D.; Bochev, I.; Ivanova-Todorova, E.; Mourdjeva, M.; Oreshkova, T.; Belemezova, K.; Kyurkchiev, S. Secretion of Immunoregulatory Cytokines by Mesenchymal Stem Cells. World J. Stem Cell. 2014, 6, 552.

(38) Jansen, K. A.; Bacabac, R. G.; Piechocka, I. K.; Koenderink, G. H. Cells Actively Stiffen Fibrin Networks by Generating Contractile Stress. Biophys. J. 2013, 105, 2240-2251.

(39) van Helvert, S.; Friedl, P. Strain Stiffening of Fibrillar Collagen During Individual and Collective Cell Migration Identified by Afm Nanoindentation. ACS Appl. Mater. Interfaces 2016, 8, 21946-21955. 